\title{
Managementgrundlagencurriculum für Assistenzärztinnen und Assistenzärzte in Spitälern
}

Das Weiterbildungscurriculum ist ein kompakter Kurs (total 6 Tage) über Grundlagen des Managements, das Zusammenspiel von Arbeitsprozessen und ärztlichen Funktionen, zur Kommunikation und Kooperation in Organisationen, Qualitätsentwicklung, rechtlichen Fragen sowie zum Selbstmanagement und zur Laufbahnplanung. Als Innovation im 2005 wird das Modul Evidence-based Medicine EBM in Form von E-Learning durchgeführt.

Das Weiterbildungscurriculum ist einzigartig und spezifisch auf Anforderungen und Bedürfnisse von Assistenzärztinnen und -ärzten mit dem Ziel einer Spitalarzttätigkeit zugeschnitten und wird vom College-M im Auftrag der FMH durchgeführt und vom VSAO unterstützt.

\section{Lernziele}

- Die wichtigsten Entwicklungen und deren Bedeutung im Gesundheitssystem kennen.

- Ein Verständnis von Management, seinen Entwicklungen, Werkzeugen und Vorgehensweisen entwickeln.

- Grundlegende Dimensionen des Führens erkennen unter Berücksichtigung gesundheitsökonomischer Aspekte und Entwicklungen.

- Die wichtigsten Qualitätskonzepte und Gesetzesgrundlagen im Gesundheitssystem kennen.

- Vertieftes Verständnis des Zusammenspiels verschiedener Funktionen und Prozesse in (Spital-)Organisationen.

- Die Bedeutung der Selbstführung und der Laufbahnplanung kennen.

- Grundlegendes Wissen um rechtliche Fragen.

\section{Teilnehmende}

Das Managementgrundlagencurriculum richtet sich an Assistenzärztinnen und -ärzte in Spitälern. Die einzelnen Curriculumgruppen bestehen aus 20-24 Teilnehmenden. Sie werden von ausgewiesenen Trainern geleitet und durch Lernpartnerschaften der Teilnehmenden unterstützt.

\section{Programmaufbau}

Das Weiterbildungscurriculum ist als Entwicklungs- und Lernprozess gestaltet und gliedert sich in zeitlich und thematisch getrennte Weiterbildungselemente. Die einzelnen Themen bzw. Elemente sind eng koordiniert und verknüpfen sich mit der beruflichen Funktion der teilnehmenden Ärztinnen und Ärzte.

\section{Programmleiter}

- Daniel Germann, Dr. med., Mitglied der Geschäftsleitung Spitalregion St. Gallen Rorschach

- Thomas Lempert, lic. theol., Psychotherapeut, Organisationsberater

- Hansjürg Lusti, dipl. Psych., Systemtherapeut, Supervisor, Organisationsberater

- Stephan Scharfenberger, Dipl. in sozialer Arbeit FH, Systemtherapeut, Supervisor, Organisationsberater

Die Seminare werden grösstenteils im Co-Teaching geführt.

\section{Daten und Ort}

\begin{tabular}{|lll} 
Element 1 & $\begin{array}{l}\text { Freitag, 26.8.2005, } \\
\text { bis Samstag, 27.8.2005 }\end{array}$ & $\begin{array}{l}\text { Bern, Basel } \\
\text { und Zürich }\end{array}$ \\
\hline Element 2 & $\begin{array}{l}\text { Freitag, 23.9.2005, } \\
\text { bis Samstag, 24.9.2005 }\end{array}$ & $\begin{array}{l}\text { Bern, Basel } \\
\text { und Zürich }\end{array}$ \\
\hline Element 3 & $\begin{array}{l}\text { Freitag, 21.10.2005, } \\
\text { bis Samstag, 22.10.2005 }\end{array}$ & $\begin{array}{l}\text { Bern, Basel } \\
\text { und Zürich }\end{array}$ \\
\hline Element 4 & $\begin{array}{l}\text { Freitag, 9.12.2005, } \\
\text { bis Samstag, 10.12.2005 }\end{array}$ & $\begin{array}{l}\text { Bern, Basel } \\
\text { und Zürich }\end{array}$
\end{tabular}

\section{Preis}

Der Unkostenbeitrag der Teilnehmenden beträgt Fr. 390.-.

\section{Informationen und Anmeldungen}

Detaillierte Informationen finden Sie unter: www.college-m.ch, College-M, Freiburgstrasse 41, 3010 Bern, Tel. 03163230 26, Fax 031632 30 25, E-Mail: info@college-m.ch. 